\title{
Who are we treating?
}

Clarification-The Analysis article by Abramson et al, which is referred to in this Editor's Choice (BMJ 2014;348:g1954, doi:10.1136/bmj.g1954) has been corrected.

Cite this as: BMJ 2014;348:g3355

๑ BMJ Publishing Group Ltd 2014 